\title{
Communication management system design of the smart grid based on the GIS
}

\author{
Zhirong Liang ${ }^{1, a}$, Qi Xue ${ }^{1, b}$ and Ying Du ${ }^{1, c}$ \\ ${ }^{1}$ Chinese people's Liberation Army Armored Forces Academy, Anhui, 233000, China \\ a476772954@qq.com, bxueqihero@163.com, '693221379@qq.com
}

Keywords: GIS; smart grid; communication management system; design

\begin{abstract}
The emergence of the smart grid has put a higher management requirement of the current electric power communication network. In response to it, people must gradually realize intelligentization and upgrade of the communication management system, apart from increasing its efficiency. Geographic Information System (GIS) could well meet the requirement. Based on the GIS, this paper should adopt corresponding technical methods to design the communication management system, aiming at improving the stability of the electric power communication network.
\end{abstract}

\section{Introduction}

GIS is the abbreviation of Geographic Information System. Its emergence has improved the safety and economy of grid operation. With the appearance and development of the smart grid, the electric power grid network is faced with more and more management demands. To meet these demands, it is necessary to design communication management system based on the GIS so as to efficiently control and manage the electric power communication network, improve its operation stability and get quickly fixed when there are faults.

\section{Demand analysis of the communication management system}

The major goal of communication management system design is to ensure the steady operation of the electric power communication network and improve the electric power communication network's fault treatment capability. In terms of the traditional electric power communication network management, once there are troubles, personnel will shoot them one by one. The trouble-shooting is time-consuming, and the trouble positioning effect is poor, which serious impedes the operation of the electric power communication network. During the operation process of the communication routes and the electric transmission routes, a large amount of data information is generated. The analysis and evaluation effect of the communication routes in the original management system is not satisfying. Concerning deficits existing in the original electric power communication network management, it is necessary to equip with the communication management system design with functions, including the communication route faults smart positioning, the route resources smart management, the GIS thematic map invoking and demonstration and the routing inspection smart management [1].

\section{Communication management system design of the smart grid based on GIS}

\section{Communication route faults smart positioning.}

First, optical cable wiring diagram faults positioning. During the operation process of the electric power communication network, optical cable faults can bring serious influences. In order to shorten the fault positioning time and quickly recover the operation of the communication network, the optical cable wiring diagram in the communication management system can be adopted for the purpose. Besides, the GIS can help accurately get the geological location of certain fault [2]. In terms of system design, the optical cable diagram is put into the system, and the corresponding keywords 
for retrieval are set. After any fault occurs, the keyword can be input and the system will automatically calculate to achieve accurate positioning of the fault.

Second, defective management flow faults positioning. Apart from the above positioning methods, defective management flow faults positioning is also a smart positioning method. The function design is similar to the method adopted by the optical cable wiring diagram. After the fault positioning conditions are put into the system, the system will display the location of the fault through invoking.

Third, demarcation point checking and analysis. The optical cable routing map cannot show changes of the mixed laying styles of the optical cable. During system design, changes of the optical cable laying styles are reflected in the form of optical cable demarcation points. Based on the GIS, demarcation point checking can be realized so as to efficiently master relevant information of the changing points.

\section{Advanced statistical analysis of communication routes.}

First, the same-route statistical analysis. Mixed laying is a common laying style for the primary circuit and the communication route. As a result, the same-route phenomenon comes into being. According to the specific length of the same-route, there are two kinds of the same-route, including the full same-route and the partial same-route. During the construction process of circuit transformation and laying, if the route situation is not accurately learned, the other circuits might be easily damaged, thus influencing the operation of the communication network. In response to problems of the kind, the same-route analysis function is set when it comes to the design of the communication management system. During the setting process, it is integrated with the GIS to improve the efficiency of statistical analysis. When the same-route of the optical cable is statistically analyzed, personnel input the name of the primary circuit into it, and then the system will display corresponding information, such as the electric power tower information, the channel information, etc. Besides, the input of the optical cable name can finish the statistical analysis of the same-route information.

Second, the statistical analysis of the optical cable's occupation rate. During the operation process, the communication network will occupy certain amount of optical cable resources. In order to learn the occupation situation, it is necessary to conduct the statistical analysis of the optical cable's occupation rate. First, the overall usage rate of fiber cores is statistically analyzed. Through the calculation of the ratio of the total fiber cores to the total capacity of fiber cores, the overall usage rate can be worked out. Based on statistics of the usage rate of the single fiber core, the occupation rate can be worked out at last.

Third, the statistical analysis of the optical cable's service status system: During system design, information corresponding to the name of the optical cable, including the fiber core number, the carrying system and the starting and ending equipment, is put into the system. During analysis, personnel only need to put in the name of the optical cable to see specific information, which greatly improves the analysis efficiency [3].

Fourth, the statistical analysis of the optical cable's service status system. In the functional module, personnel also only need to put in the name of the optical cable to achieve analysis of its service status.

\section{Communication route GIS thematic map invoking and demonstration.}

During the design of the function, the GIS thematic map module is newly added to the management system. The GIS service is invoked through the GIS portal to demonstrate the single optical cable, shaft expansion chart, etc.

\section{Route inspection smart management.}

The route inspection smart management module consists of three designs. First, the information point management design; second, the inspection process management design; third, the inspection results demonstration and statistical analysis design. Among them, the realization of the GIS is a focus of information points management. To information points data, building and modification and other operations are a major part of the management work. During the route inspection process, plan formulation, downloading and implementation and results uploading constitute the whole inspection 
process. After the inspection, personnel should conduct statistical analysis of the results. The function is realized through the statement management module on the communication data platform.

In terms of the information button management, towers or posts near the route are the installation position of major information buttons. After installation, personnel can maintain the optical cable data information related to the information buttons through the GIS. During the maintenance process, the name of the information buttons is generated automatically. Personnel choose the optical cable belong to the information button from the system. After that, the system can operate automatically and finish maintenance [4]. In terms of route inspection management, personnel finishes the plan formation in the route inspection interface. Later, the inspection plan is downloaded through the terminal. With the terminal, personnel conduct route inspection. The terminal's task interface will demonstrate the weekly inspection tasks of personnel. After entering the specific task interface, personnel can click to download certain task, and inspect according to the downloaded task. Besides, they can upload their inspection results to the system through the terminal. To demonstrate and statistically analyze the inspection results, the inspection results are first demonstrated in the system. According to the inspection results, the personnel's inspection traces are checked. Based on statistics of the inspection tasks, the completion situation is analyzed.

\section{Conclusions}

During the communication management system design of the smart grid, the GIS system is employed to improve the smart and advanced level of various functions of the management system. The ultimate goal is to significantly improve the system's management level, ensure the steady performance of the communication network, and meet the smart grid's demand for the communication network management. The communication management system design can ensure quick and accurate fault positioning, shortening of the fault recovery time and improvement of the operation reliability of the communication network even if faults occur in the communication network.

\section{References}

[1]Shi J F, Wu J H, Wang J, et al. Application of GIS-based enterprise-level integrated platform to Zhejiang power grid[J]. Electric Power Informatization, 2012, (04): 12-15.

[2]Hu Y, Jiang Y \& Guo W. Design and implementation of communication optical fiber cable centralized monitoring and management sysem for distributed network based on GIS[J]. Chian Electric Power (Technology Edition), 2015, (05): 27-32.

[3]Chen L, Yin B, Shan B L, et al. Research on the overall management and data acquisition technique of power grid GIS[J]. Bulletin of Surveying and Mapping, 2015, (10): 118-121.

[4]Luo X W. Design and development of GIS-based power grid resources management system[J]. Guangdong Technology, 2013, (10): 53-54. 